\title{
Lugares de Urbanidad Metropolitana (Lumes). Planeamiento compartido y enfrentamiento de la pseudoparticipación en la Región Metropolitana de Belo Horizonte, Brasil
}

\author{
Clarice de Assis Libânio \\ Universidad Federal de Minas Gerais, Brasil \\ Recibido: 29 de junio del 2017 / Aprobado: 10 de septiembre del 2017 \\ doi: 10.26439/limaq2018.n004.2251
}

\begin{abstract}
El Programa Lugares de Urbanidad Metropolitana (Lumes) consiste en la constitución de espacios físicos y virtuales para el planeamiento compartido. Considerando el contexto de la urbanización planetaria y los procesos de segregación socioespacial en las grandes ciudades, el programa busca la participación efectiva de los moradores en el planeamiento de las acciones en el territorio. Anhela contribuir a la generación de autonomías y de aprendizaje social, a la ampliación del sentimiento de pertenencia al territorio y a la formación de la ciudadanía metropolitana en la región.
\end{abstract}

planeamiento metropolitano, participación comunitaria, Belo Horizonte, Lugares de Urbanidad Metropolitana (Lumes), PPDI-RMBH

Places of Metropolitan Urban Life (Lumes).

Shared planning and confrontation of pseudo-participation

in the Belo Horizonte Metropolitan Region, Brazil

The Places of Metropolitan Urban Life Program (Lumes) consists of implementing physical and virtual spaces for shared planning. Considering the context of planetary urban development and the processes of sociospatial segregation in big cities, the program seeks to effectively involve the inhabitants in planning actions in the territory. It aims to contribute to generation of autonomy, generation of social learning, broadening of the feeling of belonging to the territory, and shaping metropolitan citizenship in the region.

metropolitan planning, community participation, Belo Horizonte, Places of Metropolitan Urban Life (Lumes), PPDI-RMBH 


\section{INTRODUCCIÓN}

En el contexto de la urbanización planetaria y de la formación de las metrópolis contemporáneas, las ciudades presentan un modelo centro-periferia por definición desigual, jerárquico, excluyente y segregador. Contrariamente a lo que se podría suponer, el crecimiento urbano trajo consigo el agravamiento de la pobreza y las desigualdades, incluyendo violaciones de los derechos humanos, los derechos sociales y hasta los derechos civiles.

Con el nuevo avance del neoliberalismo en las últimas décadas, el poder público (no solo en el Brasil) ha sido más que socio del capital, ha actuado en beneficio del mercado inmobiliario y ha fragilizado las políticas públicas y sociales. Como agravante, se ve el desmoronamiento de los históricos movimientos sociales, tales como sindicatos y asociaciones de pobladores, $\mathrm{y}$ el surgimiento de nuevos movimientos que, sin embargo, no han conseguido por las vías político-institucionales la consideración de sus demandas.

Romper con la segregación socioespacial en tales contextos significa, indudablemente, enfrentarse a la pseudoparticipación en las políticas públicas y garantizar que las poblaciones logren interferir en las decisiones que les afectan directamente. Con tal perspectiva, se ha buscado en la Región Metropolitana de Belo Horizonte (RMBH), Brasil, la implantación de alternativas para la participación social en el planeamiento metropolitano.

En el artículo se presenta la experiencia de los Lugares de Urbanidad Metropolitana (Lumes), programa propuesto por el Centro de Desarrollo y Planeamiento Regional (Cedeplar) de la Facultad de Economía de la Universidad Federal de Minas Gerais (UFMG), en el contexto de la elaboración del Plan Metropolitano de Desarrollo Integrado (PDDI RMBH). Además de la realización de proyectos piloto en cinco ciudades, actualmente se está implantando la propuesta de los Lumes en once municipios de la región en un proceso de revisión de los planes directores municipales y su compatibilidad con el planeamiento metropolitano.

Los Lumes fueron concebidos como espacios, físicos y virtuales, dedicados al delineamiento de propuestas compartidas para el planeamiento metropolitano. Asumen formatos y dimensiones variados, según el perfil de cada territorio, sus demandas, socios potenciales y los procesos de construcción de las intervenciones. 
$\mathrm{Su}$ concepción abarca una dimensión fisica/presencial y una dimensión virtual, mediante acciones que estimulan una intervención efectiva de las comunidades en las políticas públicas y la construcción del sentido de ciudadanía metropolitana. También, la integración de los saberes técnicoscientíficos y populares-cotidianos, la generación de autonomía y la reducción de la disparidad en la toma de decisiones, y finalmente el enfrentamiento de la desigualdad y de la segregación en las metrópolis.

El presente texto está estructurado en tres partes, además de esta introducción. En la primera, se procura dar a conocer brevemente el contexto social de las metrópolis contemporáneas y discutir al respecto. Asimismo, reflexionar sobre los procesos de segregación socioespacial y de fragilización de los derechos de los ciudadanos en las grandes ciudades, principalmente el derecho a la toma de decisiones.

En la segunda parte se presenta la experiencia del Programa Lumes, y sus proyectos, para propiciar la reflexión sobre las posibilidades de incidencia efectiva de los moradores de la $\mathrm{RMBH}$ en las políticas públicas. Además, se indican las posibles relaciones del planeamiento metropolitano con la enseñanza y la extensión universitaria.

Finalmente, la tercera y última parte intenta formular algunas conclusiones sobre la actual práctica en ejecución, así como proponer caminos y acciones futuras dirigidas a la consolidación de la ciudadanía metropolitana en sus diversas dimensiones, a través del planeamiento compartido.

\section{URBANIZACIÓN, SEGREGACIÓN SOCIOESPACIAL Y DERECHO A LA CIUDAD}

La temática del desarrollo regional integrado se insertó en el contexto de las discusiones promovidas por varios autores a mediados de la década de 1960, cuando la cuestión urbana se impuso de manera decisiva en el mundo y en el Brasil.

Tal relevancia, entre otros factores, tuvo como fundamento la nueva configuración mundial (productiva, territorial, de flujos, etcétera) que en poco más de medio siglo transformó el planeta en un ambiente mayoritariamente urbano. Si en 1960 el 34 \% de la población mundial vivía 
en áreas urbanas, en el 2014 este porcentaje llegó al 54 \% (Organización de las Naciones Unidas, 2014).

En términos absolutos, se registró un incremento de población urbana en el mundo cinco veces mayor: se pasó de 746 millones de personas en 1950 a 3 900 millones en el 2014. Hoy, más del $73 \%$ de los europeos y el $79 \%$ de los latinoamericanos están concentrados en ciudades, porcentaje que sobrepasa el $85 \%$ en el Brasil. Tal proceso se muestra creciente, con proyecciones del $66 \%$ de la población mundial viviendo en zonas urbanas en el 2050 (91\% en el caso brasileño).

A partir de este marco, "ahora que todos somos urbanos" (Fortuna, 2009, p. 84), la temática de la urbanización y de las políticas para y en las ciudades se impone de manera inequívoca, y exige que se vuelvan a pensar las prácticas y conceptos de gestión e intervención en estos territorios cada vez más urbanizados y fragmentados.

Henri Lefebvre, al analizar la ciudad que se imponía en la década de 1960, afirmó que la industrialización fue el principal proceso que rompió con el modelo y hasta la estructura de las ciudades existentes hasta entonces, basadas en las nociones de comunidad y usufructo del espacio público. Para el autor, con la ciudad industrial se instauró el llamado tejido urbano, que marcó un punto crítico en la historia de las ciudades. Se verifica un doble proceso, de explosión (despliegue, expansión del tejido urbano) e implosión (de las centralidades y de su núcleo), con la "sociedad urbana constituyéndose sobre las ruinas de la ciudad" (Lefebvre, 2001, p. 81).

Tal proceso se ha intensificado en el siglo XXI a tal velocidad e intensidad que una nueva forma urbana domina el escenario mundial: la metrópoli. Monte-Mór (2006) denominó urbanización extensiva a este proceso y señaló lo siguiente:

El campo se urbanizó rápidamente a medida que las condiciones de producción urbano-industriales, antes restringidas a las ciudades, se extendieron más allá de los límites legales del espacio urbano y ganaron la dimensión regional y hasta nacional. Al hacerlo, el tejido urbano en el cual estas condiciones se apoyaban cargó consigo el germen de la polis, de la civitas, dando origen al proceso que llamé, en otros trabajos, de urbanización extensiva. (Monte-Mór, 2006, p. 17) 
Más allá de la extensión del tejido urbano sobre los territorios antes considerados rurales, el crecimiento de las ciudades ha traído consigo el incremento de la pobreza y de las desigualdades socioespaciales intraurbanas; asimismo, cuadros graves de violación de los derechos humanos, derechos sociales y hasta civiles. Las propias políticas de incremento del consumo y de aceleración del crecimiento (desordenado) han ocasionado graves crisis urbanas relacionadas con la movilidad, la contaminación ambiental, la ampliación de la segregación, el aumento de la violencia y los riesgos asociados a los cambios climáticos.

Tales disparidades se vuelven más relevantes en las grandes metrópolis y regiones metropolitanas — como es el caso de la RMBH—, una vez que en estos lugares la expansión del tejido urbano y los procesos de periferización de la vivienda agravan las desigualdades de acceso a la vida urbana plena, desigualdades condicionadas también por la movilidad socioespacial. En tales territorios las desigualdades en los indicadores se distribuyen de manera directamente proporcional a la segregación espacial y territorial, como antes se mencionó.

Es en la perspectiva del enfrentamiento de esta problemática tan compleja que, en los últimos años, se viene proponiendo una visión integrada de los derechos (civiles, humanos, sociales) en el concepto ampliado del derecho a la ciudad. En este se rescatan los términos de Lefebvre (2001) y se adaptan al nuevo contexto de las ciudades contemporáneas, que se basan en su mayor parte en las actividades terciarias e interconectadas mundialmente por las diversas redes y flujos de comunicación, capitales, información, etcétera.

El llamado derecho a la ciudad estaría compuesto por una serie de otros derechos —múltiples, explícitos o velados, formalizados o simplemente vividos en el día a día - y sintetiza el derecho a la ciudadanía, a la participación política, a la fiesta y a la cultura, al poder y a la riqueza colectiva. Además, la reafirmación del derecho a la ciudad surge de forma más relevante cuanto mayores son las desigualdades en las condiciones de vida y de acceso a los bienes y servicios disponibles en el espacio urbano, pero no se resume a ello: las poblaciones pobres y concentradas en las periferias y en las favela son excluidas de la ciudadanía, de la fiesta, del poder y la riqueza colectiva. 
Concebir el derecho a la ciudad como una realización efectiva de lo urbano necesariamente pasa por la ruptura con la segregación socioespacial y la reducción de las desigualdades en, por lo menos, seis dimensiones (Libânio, 2014): 1) económica (empleo, ingresos, consumo y reproducción de la vida); 2) espacial/territorial (servicios urbanos, infraestructura, localización de la vivienda, desplazamientos por la ciudad y condiciones de habitabilidad en general); 3) política (derechos de ciudadanía, capacidad de los individuos para influir en las decisiones que les afectan y de construir las formas de vivir colectivamente, participación efectiva y no solo formal de las personas en las esferas de decisión); 4) simbólica (identidad, identificación, afecto, convivencia, aceptación y pertenencia del individuo a determinado grupo o territorio; barreras simbólicas, formas de apropiación del espacio y posibilidad de una vida colectiva efectiva en la ciudad); 5) relacional (información y formación de redes individuales, personales, sociales, informativas, diversas para la alteración de las relaciones sociales y de poder en la ciudad); 6) cultural (educación, información, fruición, producción cultural y acceso al bien común del conocimiento).

Más que detenerse en la concepción teórica o la construcción de un proyecto de ciudad ideal, hay que pensar, en términos prácticos, en cómo avanzar en la búsqueda de la garantía del derecho a la ciudad y en el enfrentamiento de la segregación socioespacial en las metrópolis contemporáneas. Paralelamente al entendimiento de las luchas y conquistas de los movimientos sociales, es primordial también pensar en la acción del Estado y el papel del planeamiento urbano y regional para ejecutar el derecho a la ciudad. En este sentido, hay que considerar que las políticas públicas también pueden ser transformadoras y emancipadoras, y pueden cumplir su papel en la reducción de las desigualdades, en la ampliación de la justicia social y en la liberación de los individuos en las metrópolis brasileñas.

\section{LA EXPERIENCIA DE LOS LUMES Y EL PLANEAMIENTO EN LA RMBH}

¿Será que el Estado ha dado su contribución para la superación de la segregación socioespacial en las metrópolis contemporáneas? ¿Y cuál ha sido el papel de los instrumentos de planeamiento urbano y de los planificadores públicos en este proceso? 
Para Vainer (2001), un gobierno local que, de hecho, incluya la participación ciudadana y la garantía de derechos en su actuación, y que esté comprometido con los grupos subalternos, debe tener tres objetivos centrales: a) la reducción de las desigualdades y mejora de las condiciones materiales e inmateriales de los explotados; b) la constitución de sujetos políticos colectivos, a través de la organización y lucha popular; y c) el desmontaje de los mecanismos tradicionales de poder, tales como el clientelismo, la corrupción, el intercambio de favores y la privatización de los recursos públicos. (Libânio, 2016, p. 779)

En las últimas décadas la legislación brasileña ha avanzado al incorporar las pautas y los objetivos de los movimientos sociales, especialmente en lo relativo a la lucha por el derecho a la vivienda y por la ampliación tanto del control social como de la participación en la toma de decisiones. En estos logros se citan no solo los avances obtenidos en la Constitución Federal (1988) - la Constitución ciudadana-, sino también en el Estatuto de las Ciudades (2001) y en el recientemente aprobado Estatuto de la Metrópolis (2015).

Además de los instrumentos de la política urbana, tales estatutos garantizan las instancias de participación y avanzan en dirección hacia una visión del derecho a la ciudad (más allá del derecho a la vivienda), así como a la reducción de las desigualdades socioespaciales. En el Brasil, mientras tanto, se ve que la participación de la población en los rumbos de las políticas públicas - condición esencial para hacer efectivo el derecho a la ciudad- se ha presentado como un discurso en varias esferas, especialmente en los discursos de los poderes públicos, sin que signifique que verdaderamente esté encaminado hacia la emancipación del ciudadano como sujeto de derechos. Más allá de los discursos y las legislaciones, en la práctica lo que se ve en una gran parte de los casos es la utilización de las herramientas del planeamiento público como instrumentos de exclusión y de conservación de la segregación.

Así, independientemente de las conquistas de las últimas décadas, se podría afirmar que la dimensión política del derecho a la ciudad no ha sido garantizada en el caso brasileño por la democracia representativa y su modalidad participativa ${ }^{1}$. Muchas veces, en lugar de avances, hubo una inflexión en la

1 Entre otros factores se vio la fragilización de los espacios de participación, aun antes de la agitación democrática dada por la destitución de la presidenta Dilma Roussef y de las acciones 
incidencia popular en las políticas públicas. Al mismo tiempo, a la fragilidad del derecho concedido correspondería la ampliación del derecho tomado a través de la constitución de nuevas formas de emancipación y activismo.

Dentro de este contexto se busca encontrar nuevos caminos para la transformación de las relaciones desiguales en las ciudades. En el caso metropolitano, donde no hay una unidad político-administrativa y las desigualdades intrarregionales son flagrantes, es fundamental crear mecanismos y oportunidades para "asegurar la representación de los intereses de aquellos con menos ventajas en su posicionamiento en la región metropolitana, al mismo tiempo en que se ofrece una oportunidad de participación genuina en las políticas metropolitanas" (Sosa, 2017, p. 6).

En Minas Gerais, el Gobierno metropolitano se basa actualmente en una organización institucional para la gestión y planeamiento que está compuesta por tres órganos, conforme se aprecia en la figura 1: la Asamblea Metropolitana, responsable de la definición de las macrodirectrices y la interposición de recursos a las decisiones del consejo; el Consejo Deliberativo de Desarrollo Metropolitano, que decide sobre los asuntos relacionados con el Plan Director y el Fondo Metropolitano (instrumentos de la organización destinados al planeamiento y al soporte financiero, respectivamente), así como con las funciones públicas de interés común; y la Agencia de Desarrollo Metropolitano, órgano con función ejecutiva, de soporte técnico y apoyo a los municipios.

sucesivas archivadas por el nuevo Gobierno para el retiro de los derechos constitucionales de la población, de modo que se registraron, entre otros procesos, la falta de diálogo y de respeto por las demandas de los pobladores; las prácticas meramente consultivas 0 asambleístas, sin incorporación de las demandas de la población sobre la temática en pauta 0 sin real poder de decisión; y la institucionalización de la participación en los consejos de políticas públicas vacías y con una serie de problemas estructurales y de representatividad (Avritzer, 2010). 


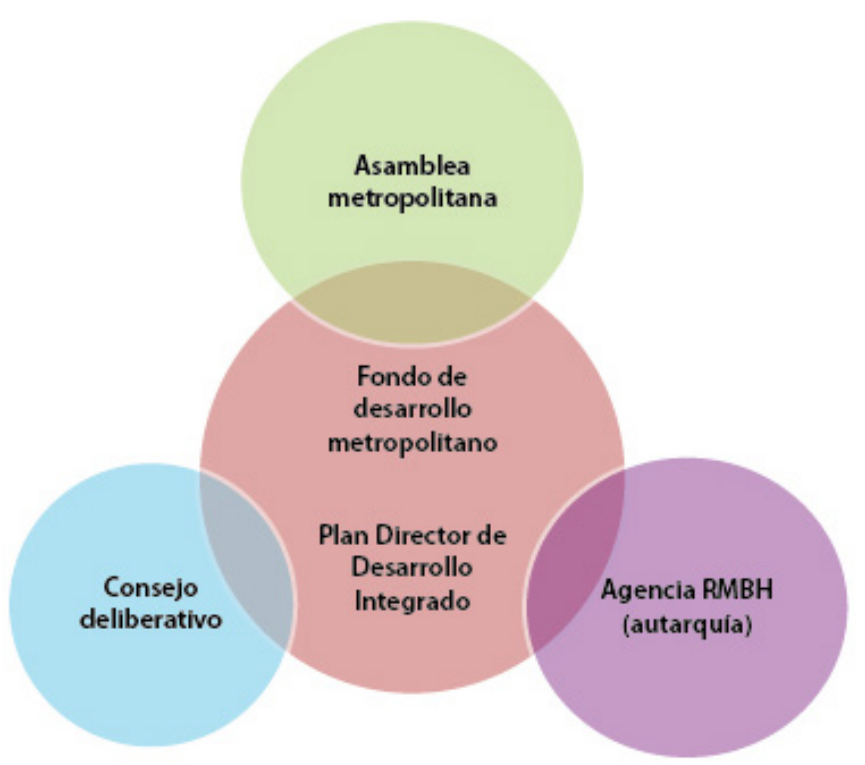

Figura 1

Organización Institucional Metropolitana de la RMBH

Fuente: Agencia de Desarrollo de la Región Metropolitana de Belo Horizonte (2017) (http://www.agenciarmbh.mg.gov.br/)

Elaboración propia

Entre el 2009 y el 2011 se elaboró el PDDI RMBH ${ }^{2}$. En forma adicional, entre el 2013 y el 2015 se desarrolló el proyecto de Macrozonificación Metropolitana (MZ RMBH), uno de los programas previstos en las 28 políticas del PDDI. En este programa se propuso definir, de acuerdo con el potencial, las características y los recursos de cada territorio, su inclinación y

2 El programa fue contratado por el Gobierno del Estado y desarrollado por el Cedeplar/UFMG, con la participación de investigadores de la Universidad Federal de Minas Gerais, la PUC Minas y la Universidad Estadual de Minas Gerais; asimismo, fue coordinado por el profesor Roberto Luis Melo Monte-Mór. 
prioridades en una organización metropolitana. De hecho, tanto el plan como el proyecto de Macrozonificación son instrumentos para que el Gobierno en sus diversos niveles pueda planear de forma articulada y sostenible el área metropolitana.

Cabe destacar que la RMBH es la tercera más grande del Brasil. Cuenta con 34 municipios $^{3}$ y cinco millones de habitantes $(26 \%$ de la población del estado), $24 \%$ del electorado y $40 \%$ del producto bruto interno del estado de Minas Gerais.

En el contexto de los proyectos antes citados, se inició la implantación de los Lumes, como forma de

organizar y difundir informaciones y conocimientos producidos en la implementación del Plan Metropolitano, sobre los programas y proyectos en ejecución y sobre las prioridades en discusión, para garantizar la participación e integración, en el sistema de planeamiento, de los diferentes agentes metropolitanos. (UFMG, 2011, p. 811)

La figura 2 muestra el mapa de la $\mathrm{RMBH}$, donde destacan los municipios que han participado hasta el momento en el programa de los Lumes.

El programa actúa transcendiendo los límites municipales y articulando las diversas escalas de poder en el territorio. Trata, al final, de profundizar en los mecanismos y procesos de participación, integración, cooperación y articulación supramunicipal, y de romper con los procesos de pseudoparticipación ${ }^{4}$

3 Ellos son Baldim, Belo Horizonte, Betim, Brumadinho, Caeté, Capim Branco, Confins, Contagem, Esmeraldas, Florestal, Ibirité, Igarapé, Itaguara, Itatiaiuçu, Jaboticatubas, Juatuba, Lagoa Santa, Mário Campos, Mateus Leme, Matozinhos, Nova Lima, Nova União, Pedro Leopoldo, Raposos, Ribeirão das Neves, Rio Acima, Rio Manso, Sabará, Santa Luzia, São Joaquim de Bicas, São José da Lapa, Sarzedo, Taquaraçu de Minas y Vespasiano.

4 Aquí se adopta el concepto utilizado por Souza (2010), tomado de Arnstein (1969), que considera varios grados de apertura del Estado para la participación popular: la no participación, en la que el Estado oprime o manipula a los ciudadanos; la pseudoparticipación, relativa a las modalidades de acción pública a través de información, consulta al ciudadano o cooptación, sin una real capacidad de influencia en la toma de decisiones; y la participación auténtica, a través de los mecanismos de asociación, delegación de poder y, en mayor grado, autogestión y autonomía de los ciudadanos en la deliberación, proposición y realización de acciones de interés colectivo. 
en el planeamiento metropolitano. Pretende contribuir al incremento del planeamiento metropolitano con acciones de democratización de la información, fortalecimiento institucional, ampliación de la participación ciudadana en la gestión del territorio y consolidación de las acciones de investigación y extensión dentro de la UFMG.

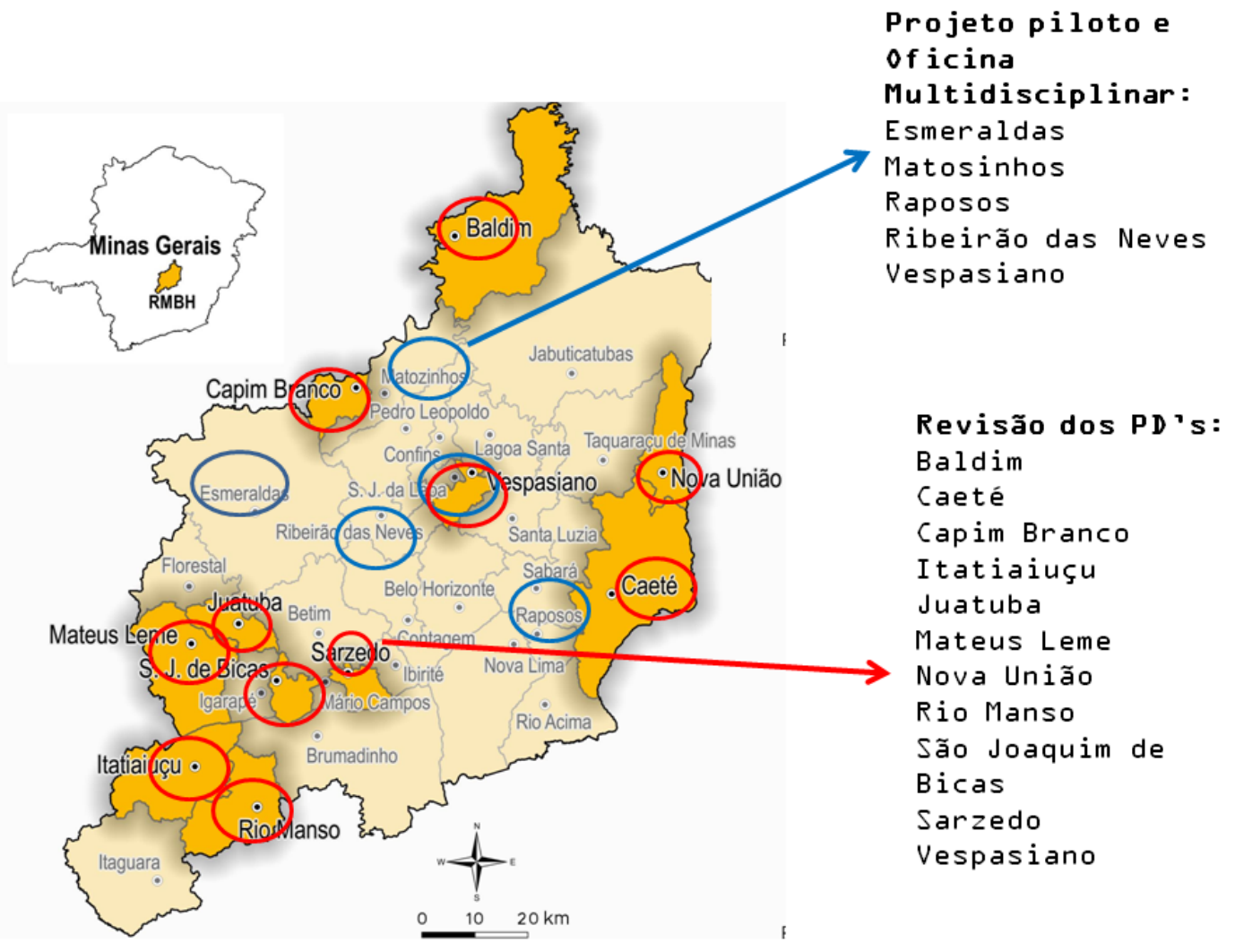

Figura 2

Mapa de la RMBH y territorios cubiertos por las acciones de los Lumes Fuente: Programa Lumes

Elaboración propia 
También destacan, entre otros, los siguientes objetivos: contribuir al delineamiento y diseminación de un sentido de identidad y ciudadanía metropolitana, y superar una visión puramente municipalista de la vida cotidiana de estos territorios; promover el intercambio de información, el diálogo y la cooperación entre los agentes metropolitanos; fortalecer la gobernanza metropolitana y la articulación entre sus diversos agentes: Gobierno del Estado, municipios, universidades y sociedad civil, bajo la coordinación de la Agencia de Desarrollo Metropolitano; ayudar en la construcción de la autonomía y de la emancipación social; formar alumnos de la UFMG en la temática interdisciplinaria del planeamiento metropolitano; y llevar el lenguaje del planeamiento a la vida cotidiana de los ciudadanos, para contribuir a una mayor incidencia popular en la toma de decisiones.

Los Lumes se estructuran y actúan en tres dimensiones y en seis programas integrados, presentados en la figura 3. Los diversos proyectos —aún en proceso de delineamiento y experimentación con los diversos actores que forman parte del planeamiento metropolitano- caminan en paralelo, cada uno con sus objetivos específicos y acciones correspondientes.

La primera dimensión es la presencial/fisica, con la instalación de espacios en los municipios para la consulta y alimentación del banco de datos, así como para el encuentro y la discusión con las comunidades. Una segunda dimensión, la virtual, es el Sistema de Informaciones, con publicación de los datos en el portal y la construcción de mapas colaborativos para una consulta ampliada. Finalmente, hay una tercera dimensión, de itinerancia y articulación interescalar, que incluye el lanzamiento de la Guía Metropolitana y la realización de cursos, procesos formativos y talleres; el fin es el fortalecimiento institucional, la formación en planeamiento metropolitano y la diseminación de las metodologías y experiencias por los municipios de la RMBH. Por encima de todas estas dimensiones está la vinculación entre enseñanza, investigación y extensión, consustanciadas en la implantación del internado metropolitano.

Desde el inicio del programa se han desarrollado algunos proyectos piloto con el objetivo de probar metodologías de acción y formar redes de asociados en el territorio. Como bien destaca Sosa (2017), no existe un modelo único para los Lumes, cada uno es formateado y desarrollado de acuerdo con el 

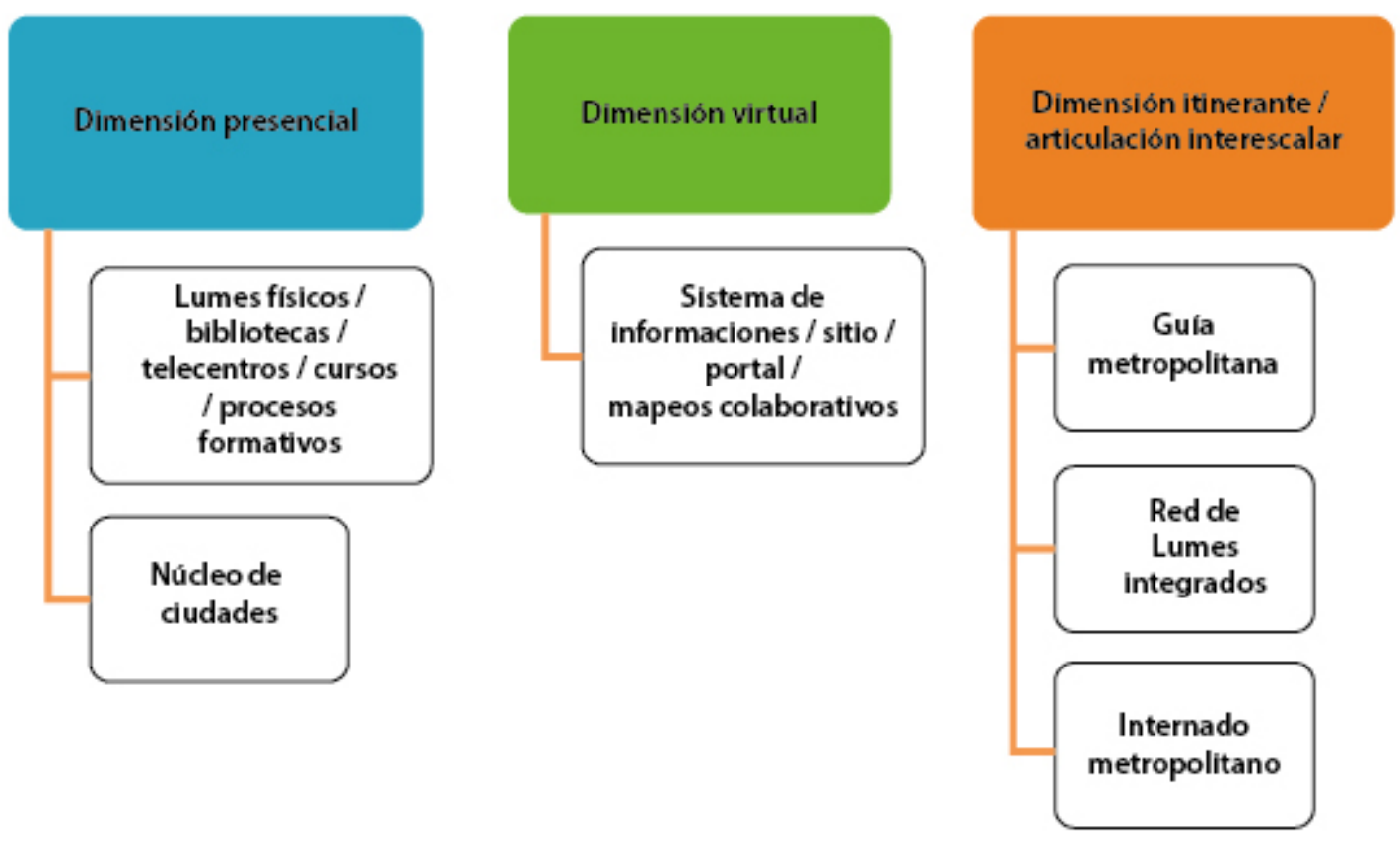

Figura 3

Diseño y funcionamiento de los Lumes

Fuente: Programa Lumes

Elaboración propia

contexto, las demandas de la comunidad y las asociaciones con los agentes sociales de los territorios, en procesos de intercambio y aprendizaje mutuos, planeamiento compartido e incorporación del "conocimiento local en la construcción de políticas metropolitanas” (Sosa, 2017, p. 16).

Se han adoptado mecanismos diversos que puedan contribuir a la generación de conocimiento y propuestas de acción bottom-up, a la divulgación de información e intercambio de saberes con la población, y a la intervención de las comunidades en las tomas de decisiones sobre su territorio. En general, se han efectuado acciones que abarcan investigaciones de campo —en las que intervienen becarios de la universidad y pobladores de las comunidades-; producción de materiales informativos; cursos, talleres y procesos formativos 
con la población y los líderes; encuentros y visitas para el intercambio de experiencias; difusión de la información y movilización comunitaria; audiencias públicas y acciones colectivas, entre otras.

Al ser imposible describir en el presente artículo todas las acciones ya desarrolladas, se optó por escoger tres de ellas para ejemplificar los caminos recorridos en la búsqueda del fortalecimiento y la concreción de la práctica del planeamiento compartido en la $\mathrm{RMBH}$.

\section{MAPA DE LA IDENTIDAD CULTURAL Y GUÍA METROPOLITANA}

Una de las principales líneas de acción del programa desde el 2014 se ha dado en el campo de la investigación social y cultural, al entenderse la importancia de la elaboración compartida de la información para las prácticas de planeamiento en el territorio, y para la identificación y formación de la ciudadanía metropolitana.

En este sentido, se está realizando el mapeo de la identidad cultural de la $\mathrm{RMBH}$ a través del levantamiento de datos en campo y vía on line. En la fase de campo se aplicaron cuestionarios y se realizaron entrevistas semiestructuradas ${ }^{5}$ con gestores de las secretarías municipales de cultura, deportes, esparcimiento, turismo, educación y desarrollo social, principalmente. Durante tal trabajo fueron mapeadas entidades, organizaciones sociales y asociaciones activas; instalaciones y servicios en el municipio; liderazgos, concejos municipales; proyectos y programas en desarrollo; calendario de fiestas locales y registro de grupos y artistas. Además, se recolectaron diagnósticos, estudios, tesis y planes municipales. Aún en la fase de campo se eligieron dos territorios para profundizar las investigaciones de la identidad cultural: el barrio de Santa Martinha, en Ribeirão das Neves, y el barrio de Santa Clara, en Vespasiano.

Tales datos fueron georreferenciados, en los casos posibles, y generaron un mapa colaborativo disponible en el sitio de internet del proyecto (ver la figura 4

5 En la primera etapa del trabajo de campo se entrevistaron a 85 gestores y líderes, fueron mapeados 926 equipamientos públicos, entidades y proyectos en los municipios; y, adicionalmente, más de 120 fiestas, 113 grupos artísticos y 224 artistas individuales, en variadas modalidades artísticas. 
e ingresar a http://www.rmbh.org.br/mapa.php). A partir de estas investigaciones, también se elaboró una matriz de análisis del perfil de los municipios de la RMBH que cuenta con tres pilares: cultura y patrimonio histórico; cultura y turismo; organización social, deporte y esparcimiento. Esto ayuda a comprender y trazar el perfil de formación histórica, y principalmente la identidad cultural de la Región Metropolitana de Belo Horizonte (Santos Soares, Rocha, Lares y Lazzarini, 2015).

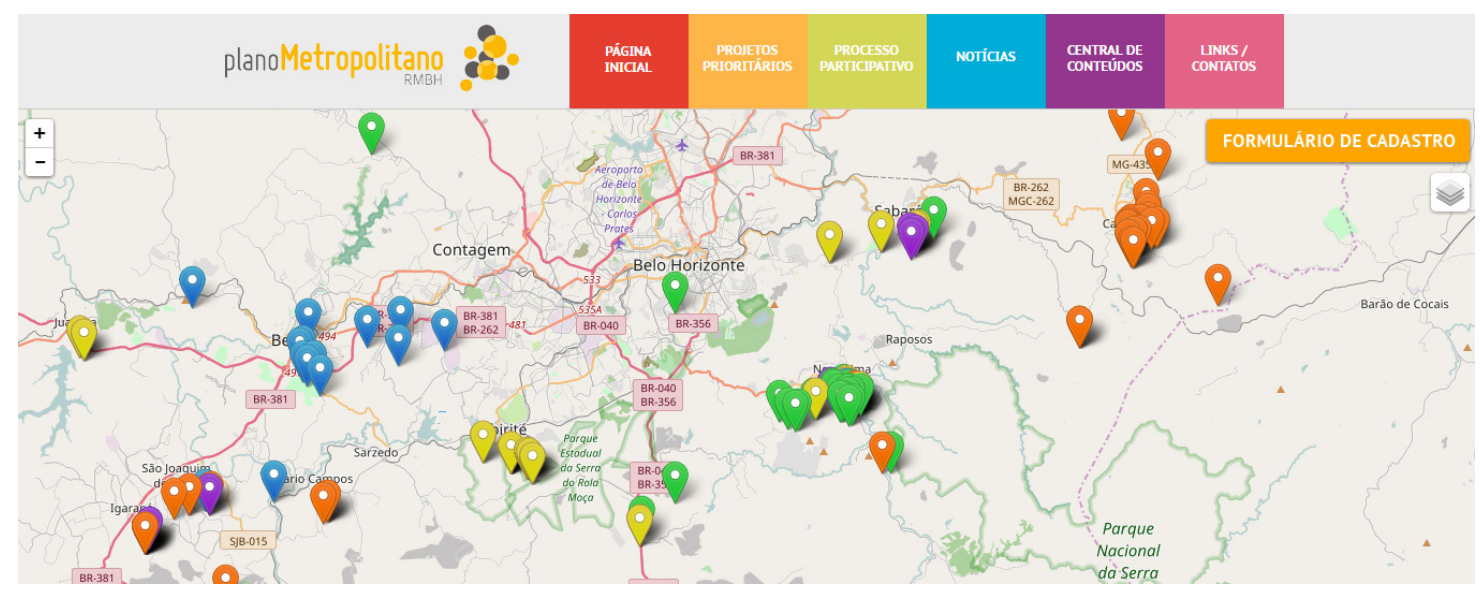

Figura 4

Mapa interactivo de la identidad cultural de la RMBH

Fuente: Plan Metropolitano (2017). Recuperado de www.rmbh.org.br

Otra acción al respecto es la Guía Metropolitana, donde se trata de mapear, conocer y diseminar las rutas culturales, ambientales y turísticas, pero principalmente los puntos de urbanidad y de interés metropolitano en el territorio de la RMBH. Con la guía, también se pretende difundir la propuesta de las zonas y los usos definidos en el proyecto $\mathrm{MZ}$ en un lenguaje accesible para los ciudadanos. Al final, se tiene la ambición de contribuir a la formación de un sentimiento de pertenencia/identificación del poblador de la RMBH con su territorio y, por tanto, a la formación de la noción de ciudadanía metropolitana. Como acciones futuras, están la continuidad del 
mapeo virtual y la investigación de campo para la Guía Metropolitana, así como su publicación digital y en medio físico.

\section{LA EXTENSIÓN UNIVERSITARIA Y EL INTERNADO METROPOLITANO}

Buscando integrar la práctica del planeamiento metropolitano en la formación de los alumnos de la universidad, el equipo de los Lumes está realizando un curso en formato de taller multidisciplinario, con carga horaria dividida entre contenidos teóricos y acción práctica. El objetivo final es crear un internado metropolitano en el que se integren enseñanza, investigación y extensión; además, que intervengan varias unidades de la UFMG en la oferta de cursos, pasantías e investigaciones en temas ligados a la ciudadanía metropolitana, planeamiento urbano participativo, gobernanza y desarrollo regional integrado.

Tres clases ya fueron ofrecidas, la cuarta está en ejecución. Se comienza con un abordaje teórico, en el que los alumnos aprenden sobre los conceptos de urbanidad,planeamiento participativo, organización metropolitana y la historia del planeamiento en la región. Seguidamente, se llevan a cabo seminarios con quienes participan en el PDDI y el MZ, y se inician los diálogos con los asociados de cada ciudad. Finalmente, los alumnos realizan investigaciones de campo, acciones de planeamiento compartido e intervención urbana al lado de los pobladores e instituciones de cada territorio.

La figura 5 contiene algunas imágenes de las acciones de campo realizadas por los alumnos y los asociados. 

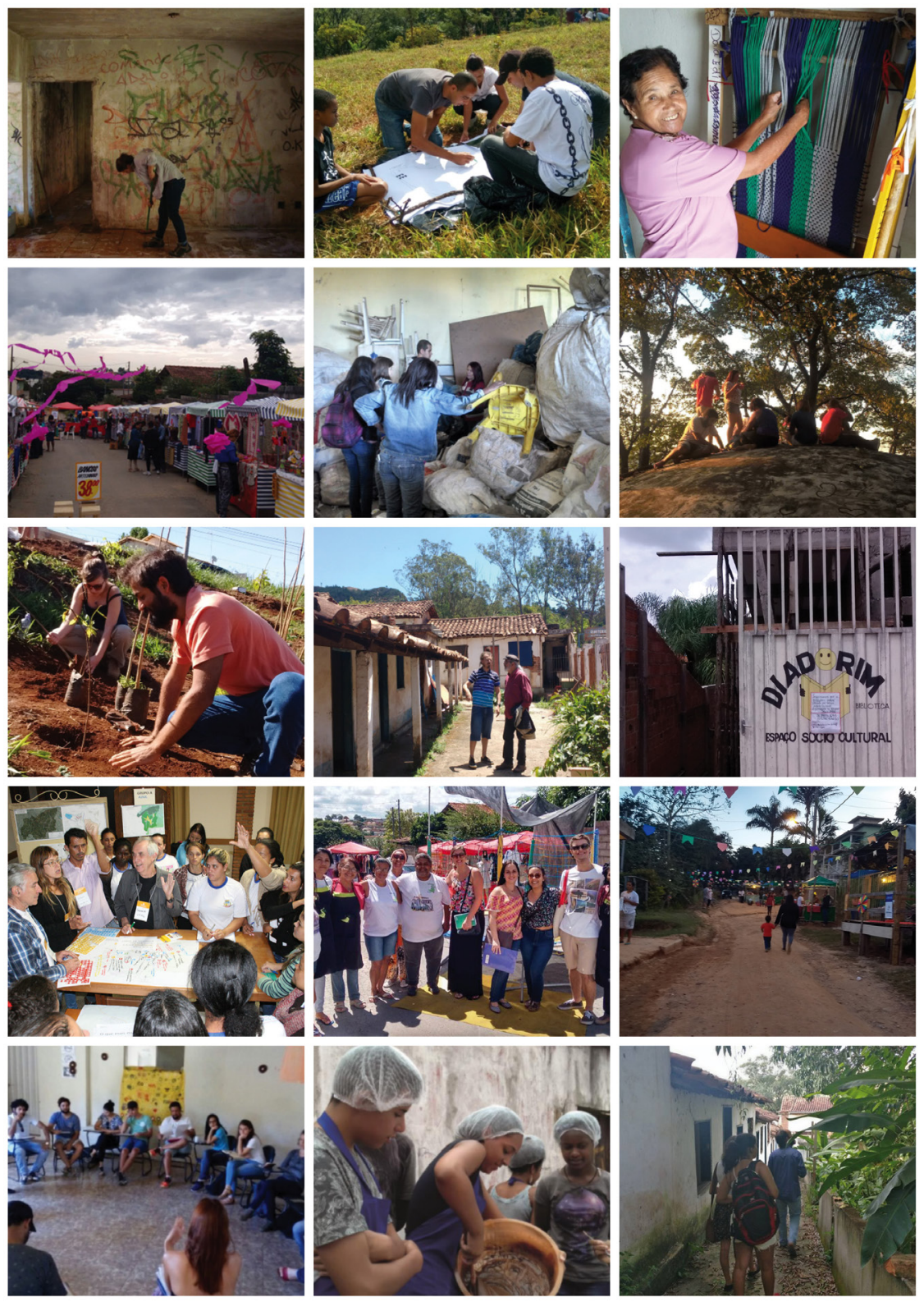

Figura 5

Acciones de extensión del internado metropolitano

Fotografías: Programa Lumes 
En total ya se han involucrado en acciones de extensión y cursos académicos casi cien alumnos de pregrado y posgrado procedentes de más de diez cursos diferentes. Ellos tuvieron una gran participación, además, relataron cuán importantes fueron la práctica y el contacto con las comunidades locales. En los tres primeros semestres se atendieron diez proyectos socioculturales de diversos municipios de la RMBH y en el cuarto semestre se ampliaron las asociaciones a otros once municipios comprendidos en el proyecto Revisión de los Planes Directores, el cual se presenta a continuación.

\section{REVISIÓN DE LOS PLANES DIRECTORES MUNICIPALES}

Es fundamental destacar la participación de los Lumes en un nuevo proyecto que desarrolla el Cedeplar/UFMG, la Revisión de los Planes Directores Municipales y su compatibilidad con el PDDI. Tal proyecto, contratado por la Agencia Metropolitana y realizado inicialmente en 11 ciudades $^{6}$ de la región, ha demostrado ser una oportunidad privilegiada para el ejercicio de la participación en el planeamiento territorial.

Para garantizar y ampliar la participación de los ciudadanos en la revisión del Plan Director, se implantaron diversos mecanismos de participación y discusión colectiva. Entre ellos destacan, más allá de las tradicionales audiencias comunitarias, la formación de un grupo de seguimiento municipal -igualitario entre la sociedad civil organizada y los Gobiernos municipal, ejecutivo y legislativo-, que lleva a cabo reuniones semanales; y la implantación del Espacio Plan Director, un ámbito, físico y virtual, donde los ciudadanos pueden buscar información, así como dar su opinión y sugerencias sobre las prioridades y necesidades de su municipio.

Considerado hoy un proyecto prioritario, ha envuelto al equipo de los Lumes y becarios en diversas actividades, especialmente en el soporte y monitoreo de los grupos de seguimiento y de sus acciones de movilización comunitaria para el planeamiento compartido. Tras casi 10 meses desde su inicio, el

6 Baldim, Caeté, Capim Branco, Itatiaiuçu, Juatuba, Mateus Leme, Nova União, Rio Manso, São Joaquim de Bicas, Sarzedo y Vespasiano. 
proceso de Revisión Participativa de los Planes Directores ha mostrado frutos muy positivos, especialmente vinculados con lo siguiente: a) la apropiación y replicación en otros territorios de las técnicas y métodos de planeamiento por las comunidades (mapas colaborativos, diagnósticos participativos, planes de acción, etcétera); b) el intercambio de saberes e información de pobladores y liderazgos de los municipios participantes con el equipo de la universidad y del Gobierno del Estado, especialmente en los talleres de lectura comunitaria y en la proposición colectiva de directrices de acción; c) la ampliación del número de personas en los encuentros de los municipios y de la UFMG, y su participación en las acciones de los Lumes; d) el reconocimiento de la importancia del planeamiento compartido en los municipios, que se traduce en un aumento en la demanda de acciones colectivas en los territorios por parte de los miembros del grupo de seguimiento municipal.

\section{CONSIDERACIONES FINALES: ¿VENTANAS PARA EL FUTURO?}

Como se vio en este artículo, el análisis y las conclusiones válidos respecto del impacto de los Lumes para hacer efectiva la participación en el planeamiento metropolitano son preliminares, dado que el programa está en proceso y, si bien es extremadamente promisorio, es embrionario aún. En los últimos años se vienen realizando sus proyectos piloto, pero aún no se puede considerar como una acción consolidada y una práctica cotidiana en el planeamiento territorial de la RMBH.

Mientras tanto, es imposible no notar que sus acciones abren muchas ventanas de posibilidades y traen indudablemente una perspectiva de participación efectiva de los pobladores de la región en la construcción compartida de las políticas metropolitanas. Desde el inicio, el programa mostró que es posible incluir a los ciudadanos en la práctica del planeamiento; también, que las personas se interesan realmente y se entusiasman cuando son llamadas para hacerlo en conjunto y para reflexionar sobre su realidad, más allá de la simple supervivencia cotidiana. En ese sentido, Sosa (2017) señala lo siguiente:

La importancia de políticas como la de los Lumes es la de permitir la participación en la escala del "espacio vivido" y la aproximación del planeamiento con los ciudadanos, lo que garantiza una mejor participación 
ciudadana y consecuentemente una mejor calidad de la democracia, siendo una escala de la disputa en la cual el ciudadano puede involucrarse de forma activa en la toma de decisiones, incentivando el fortalecimiento de una democracia metropolitana. (p. 17)

En suma, es fundamental entender que aún en forma preliminar son varios los resultados, contribuciones o avances del programa, especialmente en el sentido de: a) ampliar la voz de los sujetos/ciudadanos en la toma de decisiones sobre el espacio metropolitano; b) fomentar la práctica de la discusión de la política pública en los municipios por medio de las vivencias y experiencias concretas de planeamiento; c) provocar el sentimiento de pertenencia al territorio metropolitano en una visión más allá del municipio; d) contribuir a la creación de una cultura de participación en la RMBH; e) aproximar la estructura de la gobernanza metropolitana del día a día de los municipios a los ciudadanos; y f) formar a los alumnos de la UFMG en la temática del planeamiento metropolitano y en la práctica de la construcción compartida de programas y proyectos en el territorio.

Queda aún el desafio de garantizar que tales prácticas sean continuas y amplíen su capilaridad, su objetivo, su cobertura territorial y, por tanto, sus resultados e impactos a lo largo del tiempo. Como es un programa sin financiamiento propio, que hoy depende de recursos y becas de otros programas de la UFMG, ha tenido muchas limitaciones que necesitan ser superadas para la consolidación de las metodologías ya probadas y que mostraron su eficacia en los proyectos piloto.

Además, es fundamental avanzar y profundizar en las acciones iniciadas, con una atención especial por dos proyectos que aún no se han concretado en su plenitud: el Núcleo de Ciudades, orientado a la práctica del planeamiento compartido, al lado de los alumnos de las escuelas públicas, para crear en la base del aprendizaje una mirada colectiva hacia el territorio que forma parte de la vida cotidiana del ciudadano en formación; y la publicación y difusión de la Guía Metropolitana, una herramienta imprescindible para el conocimiento y reconocimiento del territorio, así como para la formación del sentimiento de pertenencia y de identidad metropolitana en la RMBH.

Finalmente, se espera que en el futuro de las metrópolis los procesos de planeamiento puedan contribuir a ampliar el derecho a la ciudad. También, 
que puedan enfrentarse a la pseudoparticipación de los ciudadanos en las políticas públicas y romper con ella a través de la incorporación de los procesos de producción colectiva de conocimiento, colaboración e integración intrarregional, para crear así una red metropolitana formada por los diversos agentes que actúan en este territorio tan plural.

Los Lumes son una pequeña parte de este esfuerzo recién iniciado, pero con grandes promesas para el futuro.

\section{REFERENCIAS}

Avritzer, L. (2010). Repensando os mecanismos participativos de Belo Horizonte: representação, capacitação e intersetorialidade. (Informe interno). Belo Horizonte: PROEP.

Centro de Desarrollo y Planeamiento Regional. (2011). Plano Diretor de Desenvolvimento Integrado da Região Metropolitana de Belo Horizonte - PDDI (vol. 1). Recuperado de www.rmbh.org.br/pddi/

Fortuna, C. (2009). Cidade e urbanidade. In plural de cidade: novos léxicos urbanos. Coleção CES. Coimbra: Editorial Almedina.

Lefebvre, H. (2001). O direito à cidade. São Paulo: Centauro.

Libânio, C.A. (2014). As múltiplas dimensões do acesso à cidade e o papel do capital cultural e das redes. In Anales del $13{ }^{\circ}$ Seminário Internacional da Rede Iberoamericana de Investigadores Sobre Globalização e Território en Salvador en el año de 2014. Salvador: SEI.

Libânio, C.A. (2016). O fim das favelas? Planejamento, participação e remoção de famílias em Belo Horizonte. Cadernos Metrópole, 18(37), 765-784.

Lopes-de-Souza, M. (2010). Mudar a cidade: uma introdução crítica ao planejamento e gestão urbanos. Río de Janeiro: Bertrand Brasil.

Monte-Mór, R. L. (2006). O que é o urbano no mundo contemporâneo. Manuscrito en preparación, (281). Recuperado de http://www.ipardes. pr.gov.br/ojs/index.php/revistaparanaense/article/view/58 
Organización de las Naciones Unidas. (2014). World Urbanization Prospects. Recuperado de http://esa.un.org/unpd/wup/Highlights/WUP2014Highlights.pdf

Santos Soares, M., Rocha, A., Lares, A., y Lazzarini, J. (2015). Mapeamento da identidade cultural na Região Metropolitana de Belo Horizonte e a contribuição do projeto Lumes na constituição de uma cidadania metropolitana. Manuscrito en preparación.

Sosa, M. F. (2017).Território, identidade e política: reflexões sobre os Lugares de Urbanidade Metropolitana no contexto do planejamento urbano na RMBH. En Anales del XVII Encontro da ANPUR - Desenvolvimento, Crise e Resistência: Quais os Caminhos do Planejamento Urbano e Regional? Recuperado de http://anpur.org.br/xviienanpur/principal/ publicacoes/XVII.ENANPUR_Anais/ST_Sessoes_Tematicas/ST\%20 10/ST\%2010.1/ST\%2010.1-05.pdf

Universidade Federal de Minas Gerais. (2011). Plano Director de Desenvolvimento Integrado de Região Metropolitana de Belo Horizonte. PDDI, volumen IV. Recuperado de http://www.agenciarmbh.mg.gov. br/wp-content/uploads/2016/04/PDDI-RMBH-Relat\%C3\%B3riofinal-Volume-4.pdf 